\title{
A Computational Approach for Image Fusion using Medical Images based on Ripplet Transform
}

\author{
Rajeshwari S. Goswami \\ PG Student \\ Dept. of Electronics \& Telecom \\ L.G.N. Sapkal College of Engg. Nashik
}

\author{
Seema R. Baji \\ Assistant Professor \\ Dept. of Electronics \& Telecom \\ L.G.N. Sapkal College of Engg. Nashik
}

\begin{abstract}
Image fusion is the process of combine different images from one or multiple imaging to raise the imaging quality. The concept is to improve the image content by combing images. Image fusion is important for improving the image quality and clinical application of medical image. Computed Tomography best suited for bone rift and reduction in providing information of the tissues, at the same time Magnetic Resolution Imaging gives soft tissue information and lacks in boundary information. In this paper Ripplet transform is resolving 2 dimensional singularities and show image edges more efficiently. At beginning the medical images are reconstruct by discrete ripplet transform. Fusion rules are applied to low frequency and high frequency subband. Apply inverse discrete ripplet transform to fused coefficient of low frequency and high frequency, for getting fused image. The performance of proposed system is calculated by quantitative approach such as spatial frequency, entropy, mutual information, peak signal to noise ratio, and root mean square error.
\end{abstract}

\section{Keywords}

Medical Image Fusion, Ripplet transform, spatial frequency, Entropy, Mutual information, Peak signal noise ratio, Root mean square error.

\section{INTRODUCTION}

Nowadays, in medical field researcher (doctors) require high spatial and spectral information in single image for purpose of monitoring and treatment. This sort of information cannot be achieved using individual modality images. Particular modalities of imaging reflect knowledge of organs and tissue of human body [3]. According to their application range, they have different species such as CT, MRI, PET, SPECT, and FMRI. A Computed tomography (CT scan) is best suited for viewing bone injuries, diagnosing different organ problem such as brain, lung, chest etc. An MRI is suited for soft tissue and organ. SPECT and PET are the nuclear medicine tomographic imaging technique by applying gamma rays. FMRI is a procedure that measures brain movement by detecting correlated changes in blood flow. CT and MRI provide high resolution image with biological information. Functional image such as PET, SPECT, and fMRI gives low spatial resolution with basic information. A single medical image cannot give complete and detailed information. For this situation fusion is a technique used to combine medical images from MRI, CT scanner. The aim of image fusion is integrating equivalent give redundant information from multiple images to create fused images. These fused images are more suitable for radiologist and machine approach or further image analysis task. Image fusion is used in many operations such as remote sensing, in satellite imaging, machine vision etc.

Image fusion methods have been proposed by different researcher in literature [2]-[9]. Image fusion techniques divided into unite state as pixel, feature and decision level. It has been found that pixel level IF method shows contrast reduction. Technique based on intensity hue saturation, principal component analysis and brovey transform gives good result than previous technique but go through spectral degradation. Multiresolution analysis based IF techniques have been proposed to enhance the fusion result. Pyramidal IF system having subtype as laplacian pyramid, the gradient pyramid, the contrast pyramid, and the morphological pyramid. It fails to spatial orientation selectivity hence these IF system causes blocking effect. The dilemma with wavelet transform is that it can keep the spectral information but cannot spatial aspect.

In this paper we proposed image fusion of medical image with the help of ripplet transform. The other part of paper is organized as RT in section2, in section 3we have the proposed method. Performance evaluation given in section4. Experimental Results in section 5and Conclusion is given in section 6.

\section{RIPPLET TRANSFORM (RT)}

Fourier transforms and wavelet transform suffers from discontinuation such as boundary and curve in image. Hence for this problem, jun $\mathrm{xu}$ and dapeng wu proposed a new tool called Ripplet transform. Ripplet transform is greater dimensional generalization of curvelet transform (CVT), suited for representing image or two dimensional signals in different scale and direction [11]. Anyhow RT gives us a new compact frame work with representation of image.

\subsection{Continuous Ripplet transforms (CRT)}

For 2 dimensional image $\mathrm{F}(\mathrm{y})$ and inner product of $\mathrm{F}(\mathrm{y})$ and

$$
\begin{aligned}
& \text { ripplets } \rho_{a b \theta(\mathrm{y})}^{\mathrm{r}} \underset{\mathrm{r}}{\mathrm{r}} \text { as } \\
& R(\mathrm{a}, \overline{\mathrm{b}}, \theta)=\left\langle f, \rho_{a, b, \theta}^{\mathrm{r}}\right\rangle=\int f(\mathrm{y}) \rho_{a, b, \theta}^{\mathrm{r}} \underset{\mathrm{r}}{\mathrm{r}}(\overline{\mathrm{y}} \mathrm{\textrm {r }} \\
& R(a, b, \theta) \text { is ripplet coefficient. }
\end{aligned}
$$

\subsection{Discrete Ripplet Transform (DRT)}

For digital image processing we need discrete transform than continuous transform, hence define discretization of Ripplet transform.

For image $\mathrm{M} \times \mathrm{N}$ as 
$\left.\mathrm{R} \underset{\mathrm{j}, \mathrm{k}, \mathrm{r}}{\mathrm{r}}=\sum_{\mathrm{n} 1=0 \mathrm{n} 2=0}^{\mathrm{M}-1} \sum_{\mathrm{N}-1}^{\mathrm{N}} \mathrm{f} 1, \mathrm{n} 2\right) \stackrel{\mathrm{r}}{\mathrm{j}, \mathrm{r}, 1} \mathrm{r}(\mathrm{n} 1, \mathrm{n} 2)$

The image can get back with help of inverse discrete ripplet transform

$$
f(\mathrm{n} 1, \mathrm{n} 2)=\sum_{j k} \sum_{k} \sum_{l} R{\underset{j, k}{\mathrm{r}}, l}^{\mathrm{r}} \underset{j, k, l}{\mathrm{r}}(\mathrm{n} 1, \mathrm{n} 2)
$$

\section{PROPOSED SYSTEM}

The fused image is fused well or not statistical analysis is carried out.

The medical image fusions to be registered and assure that the equivalent pixel are adjusted. The proposed method is as follows:

(i)The source images CT image as (X) and MRI image as(Y) are break down by DRT.

(ii)For next Stage the original source images breaks as low frequency and high frequency subband. These subbands are merging using different fusion rules.

(iii) By applying Fusion rule we get respective fused low frequency and high frequency subbands.

(iv)To get final fused image F, apply inverse ripplet transform to fused low frequency and high frequency subband. It is shown in figure 1

The alphabets are used as follows $\mathrm{X}, \mathrm{Y}, \mathrm{F}$ shows that source image as CT image and MRI images also fused image respectively. ${ }_{C M}^{N}$ (p) giving the subband after applying DRT. $(\mathrm{M}=\mathrm{X}, \mathrm{Y}, \mathrm{F})$ and $(\mathrm{N}=\mathrm{L}, \mathrm{H})$ where $\mathrm{L}$ and $\mathrm{H}$ are low and high frequency subband.

For fusing subband the fusion rules are as follows

\subsection{Simple Average Fusion rule:}

Simple average fusion rule gives importance to both source images i.e. CT and MRI images,

$$
\stackrel{N}{C_{F}}(\mathrm{p})=\frac{1}{2}\left(\stackrel{N}{C_{X}}(\mathrm{p})+\stackrel{N}{C}(\mathrm{p})\right)
$$

\subsection{Maximum Selection fusion rule:}

It selects the maximum frequency coefficient of the fused image.

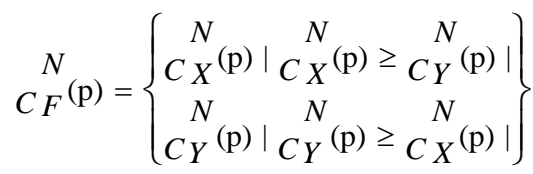

\section{PERFORMANCE EVALUTION}

The fused image is fused well or not statistical analysis is carried out.

\subsection{Standard Deviation (STD):}

Standard deviation measure the variation in the fused image. An image with high variation will have a high STD.

$$
\mathrm{STD}=\sqrt{\frac{1}{M N} \sum_{m=1}^{M} \sum_{n=1}^{N}((F(\mathrm{~m}, \mathrm{n})-\text { mean }) 2}
$$

$$
\text { mean }=\frac{1}{M N} \sum_{m=1}^{M} \sum_{n=1}^{N}|\mathrm{~F}(\mathrm{~m}, \mathrm{n})|
$$

\subsection{Entropy (EN):}

It measures information of image content. If entropy of fused image higher than input image i.e. means it have more information.

$$
E N=-\sum_{g=0}^{L-1} p(\mathrm{~g}) \log _{2} p(\mathrm{~g})
$$

$\mathrm{P}$ (g) is probability of grey level $\mathrm{g}$, and its range from $[0, \ldots \ldots \ldots$.L-1].

\subsection{Spatial Frequency (SF):}

SF can used to calculate activity and clarity level of an image. Greater the spatial frequency value gives better fusion result.

$S F=\sqrt{R F^{2}+C F^{2}}$

Row frequency denoted by RF and column frequency denoted by $\mathrm{CF}$

$$
\begin{aligned}
& R F=\sqrt{\frac{1}{M(\mathrm{~N}-1)} \sum_{m=0}^{M-1} \sum_{n=0}^{N-2}(\mathrm{~F}(\mathrm{~m}, \mathrm{n}+1)-\mathrm{F}(\mathrm{m}, \mathrm{n}))^{2}} \\
& C F=\sqrt{\frac{1}{(\mathrm{M}-1) \mathrm{N}} \sum_{m=0}^{M-2} \sum_{n=0}^{N-1}(\mathrm{~F}(\mathrm{~m}+1, \mathrm{n})-\mathrm{F}(\mathrm{m}, \mathrm{n}))^{2}}
\end{aligned}
$$

\subsection{Mutual Information (MI):}

MI measures the degree of dependency of the two images. Greater the measure means better quality.

$$
M I=I\left(\mathrm{x}_{X} ; \mathrm{x}_{F}\right)+I\left(\mathrm{x}_{Y} ; \mathrm{x}_{F}\right)
$$

\subsection{Root Mean Square Error (RMSE):}

Root mean square error between the reconstructed image and the original image presents error of mean intensity of image[12]

The RMSE is stated as,

$$
R M S E=\sqrt{\frac{1}{(\mathrm{M} \times N)}\left[\sum X \sum Y\left(I_{\text {true }}(X, Y){ }^{-} I_{\text {fused }}(X, Y)\right)\right]}(13)
$$

$\mathrm{I}_{\text {true }}(\mathrm{X}, \mathrm{Y})$ is reference image, $\mathrm{I}_{\text {fused }}(\mathrm{X}, \mathrm{Y})$ is fusion image also $\mathrm{M}, \mathrm{N}$ are dimension of images.

\subsection{Peak Signal to Noise Ratio (PSNR):} It given by,

$$
P S N R=10 * \operatorname{LOG} 10\left(\frac{M \times N}{R M S E^{2}}\right)
$$

$\mathrm{F}(\mathrm{m}, \mathrm{n})$ is gray value of pixel of image $\mathrm{F}$ at position $(\mathrm{m}, \mathrm{n})$ 


\section{EXPERIMENTAL RESULTS}

Table 1 Parametric analysis of Source Images

\begin{tabular}{|c|c|c|c|c|c|c|}
\hline $\begin{array}{l}\text { Sr. } \\
\text { No }\end{array}$ & System & $\begin{array}{l}\text { Image } \\
\text { No. }\end{array}$ & $\mathrm{SF}$ & S TD & ENTROPY & MI \\
\hline \multirow[t]{4}{*}{1} & existing & c1 & 2.78 & 30.04 & 0.68 & 4.29 \\
\hline & & $\mathrm{m} 1$ & 3.75 & 33.78 & 3.07 & 5.54 \\
\hline & proposed & $\mathrm{c} 1$ & 4.91 & 32.04 & 2.73 & 6.58 \\
\hline & & $\mathrm{m} 1$ & 5.98 & 35.78 & 5.25 & 7.87 \\
\hline \multirow[t]{4}{*}{2} & existing & $\mathrm{c} 2$ & 3.96 & 43.59 & 0.99 & 6.23 \\
\hline & & $\mathrm{m} 2$ & 9.09 & 81.77 & 7.43 & 13.40 \\
\hline & proposed & $c 2$ & 6.14 & 45.59 & 3.04 & 8.51 \\
\hline & & $\mathrm{m} 2$ & 11.31 & 83.77 & 9.62 & 15.73 \\
\hline \multirow[t]{4}{*}{3} & existing & c3 & 7.71 & 84.76 & 1.93 & 12.11 \\
\hline & & $\mathrm{m} 3$ & 4.30 & 38.73 & 3.52 & 6.35 \\
\hline & proposed & c3 & 9.89 & 86.76 & 3.97 & 14.39 \\
\hline & & $\mathrm{m} 3$ & 6.53 & 40.73 & 5.70 & 8.68 \\
\hline \multirow[t]{4}{*}{4} & existing & $c 4$ & 6.65 & 73.17 & 1.66 & 10.45 \\
\hline & & $\mathrm{m} 4$ & 6.52 & 58.71 & 5.34 & 9.62 \\
\hline & proposed & c4 & 8.83 & 75.17 & 3.71 & 12.74 \\
\hline & & $\mathrm{m} 4$ & 8.75 & 60.71 & 7.52 & 11.95 \\
\hline \multirow[t]{4}{*}{5} & existing & $c 5$ & 5.36 & 58.97 & 1.34 & 8.42 \\
\hline & & $\mathrm{m} 5$ & 0.47 & 4.19 & 0.38 & 0.69 \\
\hline & proposed & c5 & 7.54 & 60.97 & 3.39 & 10.71 \\
\hline & & $\mathrm{m} 5$ & 2.69 & 6.19 & 2.56 & 3.01 \\
\hline \multirow[t]{4}{*}{6} & existing & $c 6$ & 2.59 & 28.46 & 0.65 & 4.07 \\
\hline & & m6 & 8.25 & 74.27 & 6.75 & 12.18 \\
\hline & proposed & $c 6$ & 4.77 & 30.46 & 2.69 & 6.35 \\
\hline & & $\mathrm{m} 6$ & 10.47 & 76.27 & 8.93 & 14.50 \\
\hline
\end{tabular}

Here for parametric analysis of source images first consider as six images of CT and MRI images. They are having mainly existing system and proposed system. For existing system we are having contourlet method where as for proposed one ripplet method. Table 1 shows better value for source images in proposed system compare to existing one.

For table 2 the parametric analysis of fused image is shown .Here fused image no is denoted as F. In the proposed system we are calculating root mean square error (RMSE) and peak signal to noise ratio (PSNR).Here in fig 2 image set 3 and 5 shown in below

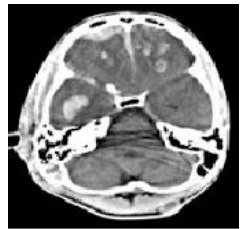

(a)

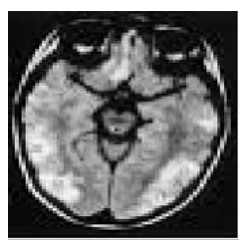

(d)

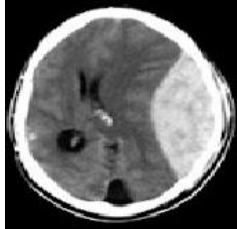

(b)

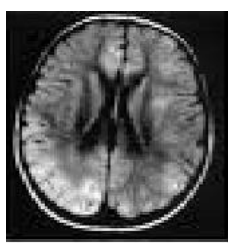

(e)

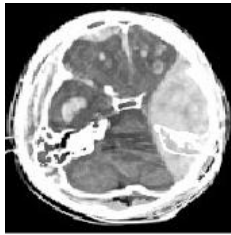

(c)

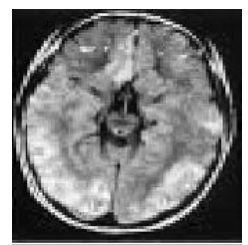

(f)
Fig.2 image (a), (d) are CT images (b), (e) are MRI images and (c) and (f) are respective fused image
Table 2 Parametric analysis of fused images

\begin{tabular}{|l|l|l|l|l|l|l|l|l|}
\hline $\begin{array}{l}\text { Sr } \\
\text { no }\end{array}$ & System & $\begin{array}{l}\text { Image } \\
\text { no }\end{array}$ & SF & STD & EN & MI & RMSE & PSNR \\
\hline 1 & existing & F1 & 5.09 & 45.84 & 4.58 & 2.18 & - & - \\
\hline & proposed & & 7.32 & 47.84 & 6.78 & 4.28 & 133.240 & 15.77 \\
\hline 2 & existing & F2 & 5.07 & 45.61 & 4.56 & 2.17 & - & - \\
\hline & proposed & & 7.29 & 47.61 & 6.76 & 4.27 & 265.793 & 18.32 \\
\hline 3 & existing & F3 & 9.29 & 83.63 & 8.36 & 3.98 & - & - \\
\hline & proposed & & 11.51 & 85.63 & 10.56 & 6.08 & 246.469 & 13.22 \\
\hline 4 & existing & F4 & 4.15 & 37.31 & 3.73 & 1.78 & - & - \\
\hline & proposed & & 6.37 & 39.31 & 5.93 & 3.87 & 254.469 & 12.68 \\
\hline 5 & existing & F5 & 7.24 & 65.18 & 6.52 & 3.10 & - & - \\
\hline & proposed & & 9.46 & 67.18 & 8.72 & 5.20 & 78.230 & 11.11 \\
\hline 6 & existing & F6 & 4.13 & 37.14 & 3.71 & 1.77 & - & - \\
\hline & proposed & & 6.35 & 39.14 & 5.91 & 3.86 & 170.894 & 20.95 \\
\hline
\end{tabular}

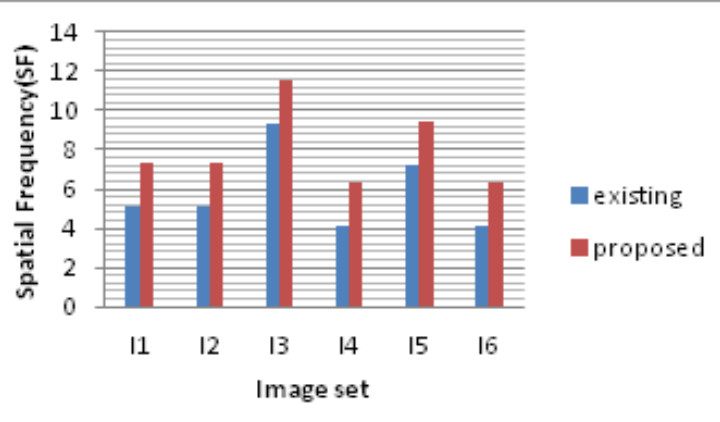

Graph 1.Analysis of spatial frequency of fused image

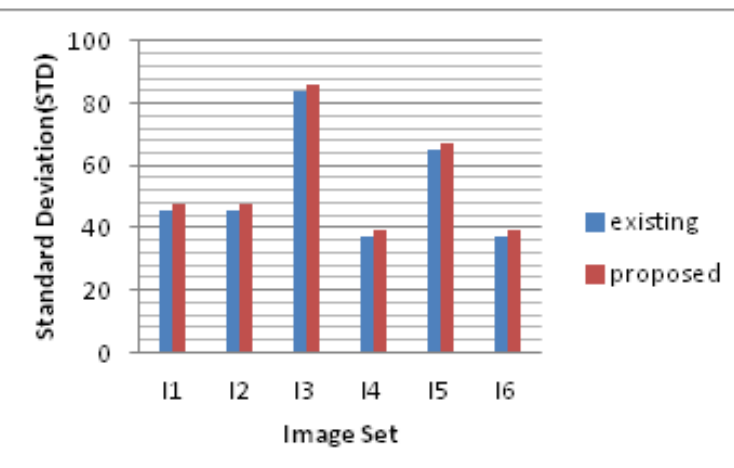

Graph2. Analysis of standard deviation of fused image

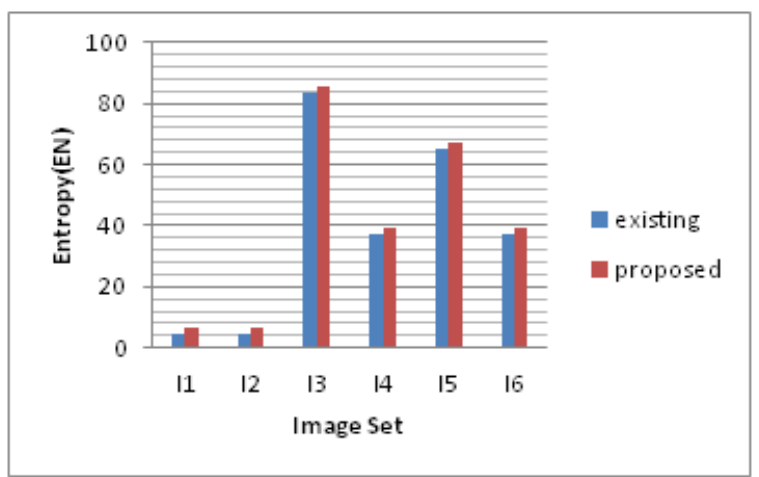

Graph3 .Analysis of Entropy of fused image

Entropy of an image is the average numbers of bits need to quantize the intensities of image. The entropy of an image is measure of information content. Analysis of entropy is shown in Graph3.In Graph4 for MI analysis shown ,MI is measures of degree of dependence. 


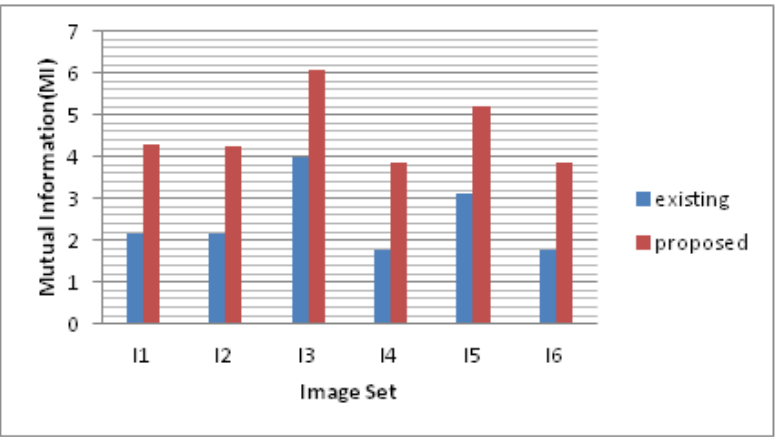

Graph 4. Analysis of mutual information of fused image

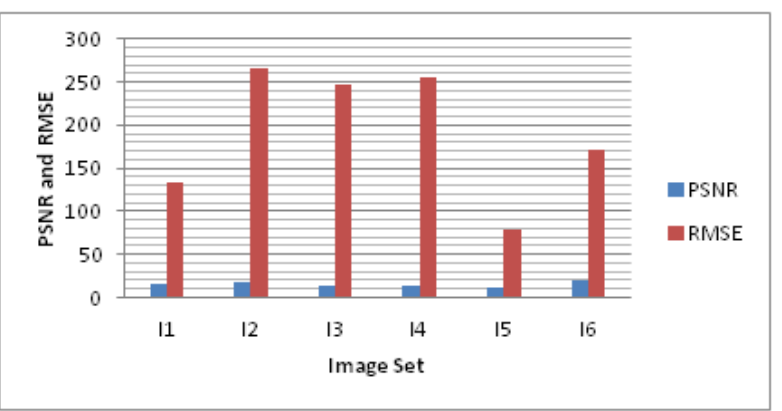

Graph5. Analysis of Peak signal to noise ratio and Root mean square error of fused image

\section{CONCLUSION}

The fusion of medical images plays a necessary role in many clinical applications. CT and MRI image subband are fused using different fusion rule. Image fusion using ripplet transform support more extensive and accurate information than individual source image. Proposed system shows higher value of spatial frequency, standard deviation, entropy, mutual information, root mean square error, and peak signal to noise ratio parameters for $\mathrm{CT}$ and MRI images as compared to existing system. Now for these system we used mainly brain images, we can analysis of other human body part such as abdomen, chest etc.

\section{Acknowledgements}

The author would like to thank the guide and the staff member of Dept. of EC from L.G.N. Sapkal college anjaneri. For their contribution and unconditional support at various stages of project. Author would like thank to civil hospital and Sai high-tech diagnostic center for providing medical images.

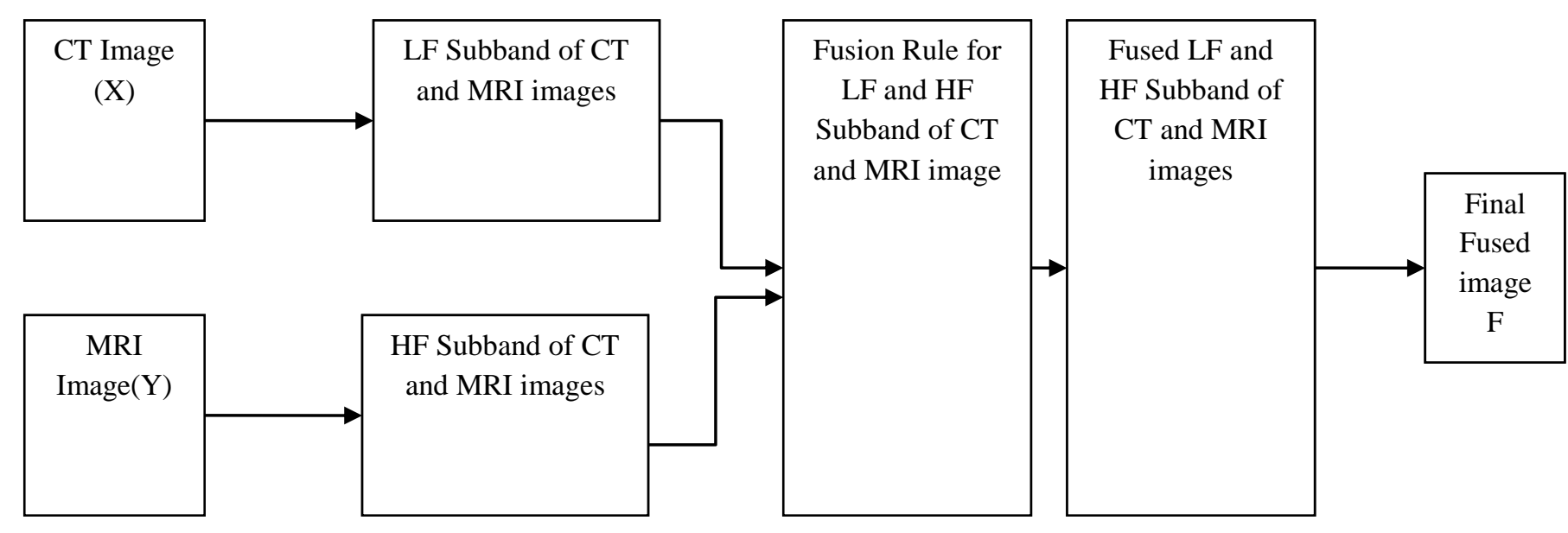

Fig .1 Proposed block diagram

\section{REFERENCES}

[1] S. T. Shivappa, B. D. Rao, M. M. Trivedi, "An Iterative decoding algorithm for fusion of multimodal information", EURASIP journal on advances in signal processing, Vol.10, pp.1-10, 2008.

[2] S. Das, M. Choudhary, M. K. Kundu, "Brain MR image classification using multiscale geometric analysis of ripplet", Progress in electromagnetic research, Vol.137,pp.1-17,jan13.

[3] J. zhang, "Multiscale remote sensing data fusion, status and trends", International Journal of image and data fusion, Vol.1,no-1,pp.5-24,march2013.

[4] H. Li, B. S. Manjunath, S. K. Mitra, "Multisensor image fusion using wavelet transform", IEEE signal processing, Vol-94, pp.51-54, 1994.
[5] D. K. Sadhu , M. P. Prasai," Different Image fusion Technique-A Critical Review", International journal of modern engineering research(IJMER),ISSN-22496645,Vol-2,Issue-5,pp.4298-4301,Oct12.

[6] M. Pradeep, "Implementation of image fusion algorithm using matlab", IEEE signal processing, pp.165-168,2013.

[7] F. E. Ali, M. E. Donkany ,A. A. Saad, "Curvelet transform for MR and CT images", Progress in electromagnetic Research, Vol.-25,pp.215-224,2010.

[8] J. L. Strck, E .J. Cande, D. L. Donoho, "The curvelet transform for image denoising ",IEEE Transcation on image processing Vol.-11,pp.670-685,june2002.

[9] S. Das, M. K. Kundu, "Ripplet based multimodality medical image fusion using pulse coupled neural and modified spatial frequency", IEEE international 
conference on recent trends in information system, pp.229-234, 2011.

[10] S. Mahajan, A. Singh, "A Comparative analysis of different image fusion technique" International journal of computer science (IIJCS), ISSN-2321-5992, Vol.-2, issue-1, pp.8-15, jan14.
[11] S. Das, M. K. Kundu, "Medical image fusion using ripplet transform type-1", Progress in electromagnetic research B, Vol.-30,pp.355-370,2011.

[12] K. Parmar, R. Kher, "A Comparative analysis of multimodality medical image fusion methods ", IEEE6th asia modeling symposium, 2012, pp93-97, 2012.

[13] J. Xu, L. Yang, D. Wu, "Ripplet A New Transform for ImageProcessing",Elsevier,Vol.-3,pp.1-24,2010. 